\title{
How to Measure Subdiffusion Parameters
}

\author{
T. Kosztołowicz ${ }^{1}$, K. Dworecki ${ }^{1}$, and St. Mrówczyński ${ }^{1,2}$ \\ ${ }^{1}$ Institute of Physics, Świętokrzyska Academy, ul. Świętokrzyska 15, PL - 25-406 Kielce, Poland \\ ${ }^{2}$ Sottan Institute for Nuclear Studies, ul. Hoża 69, PL - 00-681 Warsaw, Poland
}

(11-th April 2005)

\begin{abstract}
We propose a method to measure the subdiffusion parameter $\alpha$ and subdiffusion coefficient $D_{\alpha}$ which are defined by means of the relation $\left\langle x^{2}\right\rangle=\frac{2 D_{\alpha}}{\Gamma(1+\alpha)} t^{\alpha}$ where $\left\langle x^{2}\right\rangle$ denotes a mean square displacement of a random walker starting from $x=0$ at the initial time $t=0$. The method exploits a membrane system where a substance of interest is transported in a solvent from one vessel to another across a thin membrane which plays here only an auxiliary role. We experimentally study a diffusion of glucose and sucrose in a gel solvent, and we precisely determine the parameters $\alpha$ and $D_{\alpha}$, using a fully analytic solution of the fractional subdiffusion equation.
\end{abstract}

PACS numbers: 05.40.-a, 66.10.-x

Subdiffusion occurs in various systems. We mention here a diffusion in porous media or charge carriers transport in amorphous semiconductors $[1,2]$. The subdiffusion is characterized by a time dependence of the mean square displacement of a Brownian particle. When the particle starts form $x=0$ at the initial time $t=0$ this dependence in a one-dimension system is

$$
\left\langle x^{2}\right\rangle=\frac{2 D_{\alpha}}{\Gamma(1+\alpha)} t^{\alpha}
$$

where $D_{\alpha}$ is the subdiffusion coefficient measured in the units $\left[\mathrm{m}^{2} / \mathrm{s}^{\alpha}\right]$ and $\alpha$ obeys $0<\alpha \leq 1$. For $\alpha=1$ one deals with the normal or Gaussian diffusion characterized by the linear growth of $\left\langle x^{2}\right\rangle$ with $t$ which results from the Central Limit Theorem applied to many independent jumps of a random walker. The anomalous diffusion occurs when the theorem fails to describe the system because the distributions of summed random variables are too broad or the variables are correlated to each other. The subdiffusion is related to infinitely long average time that a random walker waits to make a finite jump. Then, its average displacement squared, which is observed in a finite time interval, is suppressed.

The subdiffusion has been recently extensively studied, see e.g. [1-4]. While the phenomenon is theoretically rather well understood there are very a few experimental investigations. There is no effective method to experimentally measure $\alpha$ and $D_{\alpha}$. In the pioneering study [4], where $D_{\alpha}$ was determined experimentally for the first time, the interdiffusion of heavy and light water in a porous medium was observed by means of NMR. $D_{\alpha}$ was found, using the special case $\alpha=2 / 3$ solution of the subdiffusion equation. The procedure is neither very accurate nor of general use.

Our aim here is to present a method to precisely measure $\alpha$ and $D_{\alpha}$. The method is described in detail in [5], here we give a brief account of it. For practical reasons, we choose for the experimental study a membrane system containing two vessels with a thin membrane in between which separates the initially homogeneous solute of the substance of interest from the pure solvent. A schematic view of the system is presented in Fig. 1. The membrane does not affect values of investigated parameters. Instead of the mean square displacement (1), our method refers to the temporal evolution of the thickness $\delta$ of the so-called near-membrane layer which is defined as a distance from the membrane where the substance concentration $C(x, t)$ drops $\kappa$ times with respect to the membrane surface i.e.

$$
C(\delta, t)=\kappa C\left(0^{+}, t\right),
$$

where $x=0$ is the position of a thin membrane and $\kappa$ is an arbitrary number smaller than unity; we used $\kappa=$ $0.12,0.08$ and 0.05 .

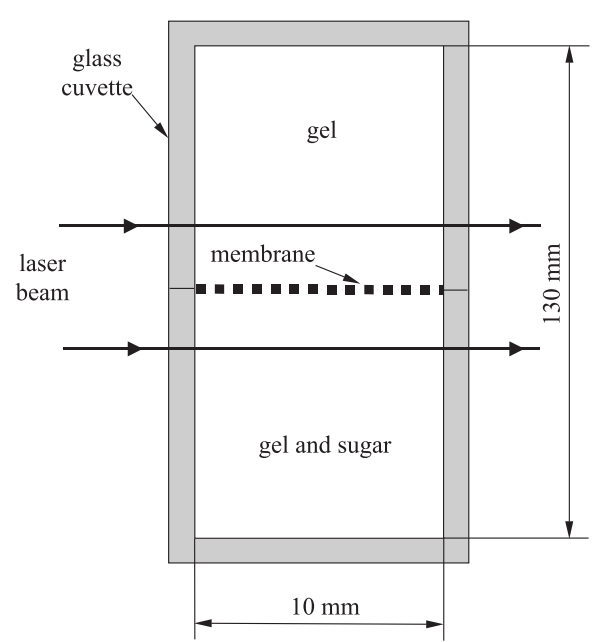

FIG. 1. Schematic view of the membrane system under study.

In our previous paper [6], we demonstrated that $\delta(t)=$ $A \sqrt{t}$ for the normal diffusion. Studying experimentally the diffusion of glucose and sucrose in a gel solvent, we show here that $\delta(t)=A t^{\gamma}$ with $\gamma<0.5$. A gel is built of large and heavy molecules which form a polymer network. Thus, the gel water solvent resembles a porous material filled with water. Since a mobility of sugar molecules is highly limited in such a medium the subdiffusion is expected. 
For each measurement, we prepared two gel samples: the pure gel - $1.5 \%$ water solution of agarose and the same gel dripped by the solute of glucose or sucrose. The concentration of both sugars in the gel was fixed to be either $0.1\left[\mathrm{~mol} / \mathrm{dm}^{3}\right]$ or $0.07\left[\mathrm{~mol} / \mathrm{dm}^{3}\right]$ but our results appear to be independent of the initial concentration. The two vessels of the membrane system were then filled with the samples and the (slow) processes of the sugar transport across the membrane started. Since the concentration gradient was in the vertical direction only, the diffusion is expected to be one-dimensional. We used an artificial membrane of the thickness below $0.1 \mathrm{~mm}$. The membrane was needed for two reasons. It initially separated the homogeneous sugar solute in one vessel from the pure gel in another one. It also precisely fixed the geometry of the whole system.

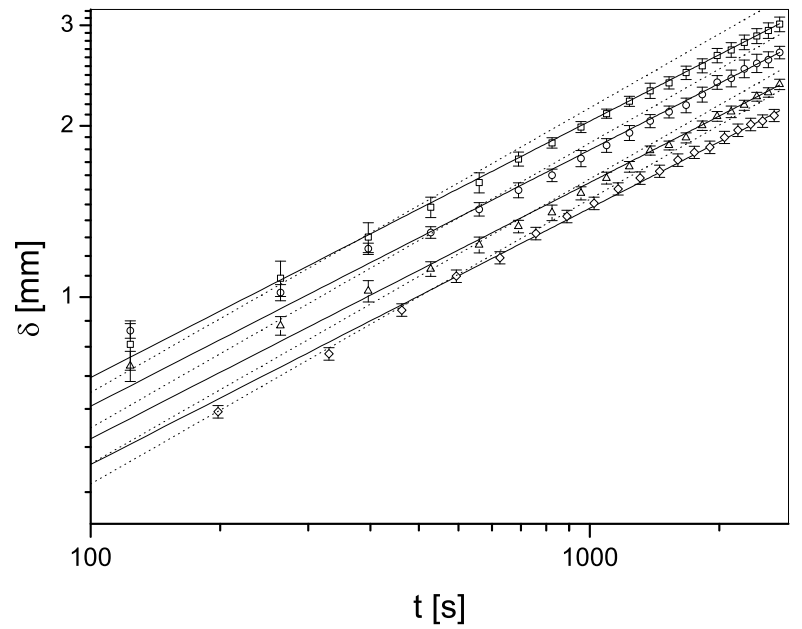

FIG. 2. The experimentally measured thickness of the near-membrane layer $\delta$ as a function of time $t$ for glucose with $\kappa=0.05(\square), \kappa=0.08(\circ), \kappa=0.12(\triangle)$, and for sucrose with $\kappa=0.08(\diamond)$. The solid lines represent the power function $A t^{0.45}$ while the dotted ones correspond to the function $A \sqrt{t}$.

The sugar concentration was measured by means of the laser interferometric method. The laser light was split into two beams. The first one went through the system parallelly to the membrane surface while the second (reference one) went directly to the light detecting system. The interferograms, which appear due to the interference of the two beams, are controlled by the refraction coefficient of the solute which is turn depends on the substance concentration. The analysis of the interferograms allows one to reconstruct the time-dependent concentration profiles of the substance transported in the system and to find the time evolution of the near-membrane layers which are of our main interest here. The experimental set-up is described in detail in [7]. It consists of the cuvette with membrane, the Mach-Zehnder interferometer including the He-Ne laser, TV-CCD camera, and the computerized data acquisition system.
When the sugar was diffusing across the membrane we were recording the concentration profiles in the vessel which initially contained pure gel. The examples of typical interferograms and extracted concentration profiles are presented in [7]. The thickness of a near-membrane layer $\delta$ was calculated from the measured concentration profiles $C(x, t)$ according to the definition (2), and thus the thickness of near-membrane layer as a function of time was found.

In Fig. 2 we present $\delta(t)$ for the glucose and sucrose of initial concentration $0.1\left[\mathrm{~mol} / \mathrm{dm}^{3}\right]$. The analysis of errors, in particular those shown in Fig. 2, is described in [5]. For the glucose we present $\delta(t)$ for three values of $\kappa=0.12,0.08$ and 0.05 while for the sucrose $\kappa=0.08$. As seen, the time dependence of $\delta$ is well described by the power function $A t^{\gamma}$ with the common index $\gamma=$ 0.45. The lines representing $\sim \sqrt{t}$ are also shown for comparison. It is evident that the measured index $\gamma$ is smaller than 0.5 . There are some deviations of our data from $A t^{0.45}$ at $t<300$ s but our final theoretical formulas, in particular the power law behavior, hold in the long time approximation.

Fitting the experimental data shown in Fig. 2, we found the universal index $\gamma=0.45 \pm 0.005$ and the parameter $A$ which depends on $\kappa$; for glucose $A=0.091 \pm 0.004$ when $\kappa=0.05, A=0.081 \pm 0.004$ when $\kappa=0.08$, and $A=0.071 \pm 0.004$ when $\kappa=0.12$; for sucrose $A=0.064 \pm 0.003$ when $\kappa=0.08$. In each case $\chi^{2}$ per degree of freedom was smaller than 1 .

The subdiffusion is described by the equation with fractional derivative $[1,8]$

$$
\frac{\partial C(x, t)}{\partial t}=D_{\alpha} \frac{\partial^{1-\alpha}}{\partial t^{1-\alpha}} \frac{\partial^{2} C(x, t)}{\partial x^{2}},
$$

which for $\alpha<1$ corresponds to an infinitely long average waiting time of the random walker - the physical situation in a gel solvent resembling the porous medium. We solve Eq. (3) in the region $x>0$ with the initial condition $C(x, 0)=C_{0}$ for $x<0$ and $C(x, 0)=0$ for $x>0$. In fact, we solve Eq. (3) for the Green's function $G\left(x, t ; x_{0}\right)$ satisfying the initial condition $G\left(x, t=0 ; x_{0}\right)=\delta\left(x-x_{0}\right)$, and then, $C(x, t)$ is calculated using the formula

$$
C(x, t)=\int G\left(x, t ; x_{0}\right) C\left(x_{0}, 0\right) d x_{0} .
$$

To find $G\left(x, t ; x_{0}\right)$ we use the relation [5]

$$
G\left(x, t ; x_{0}\right)=\int_{0}^{t} d t^{\prime} J\left(0^{+}, t^{\prime} ; x_{0}\right) G_{\mathrm{ref}}\left(x, t-t^{\prime} ; 0^{+}\right),
$$

where $x>0$ while $x_{0}<0 ; J\left(x, t ; x_{0}\right)$ is the flux associated with $G\left(x, t ; x_{0}\right)$ which for $x=0$ gives the flow across the membrane; $G_{\text {ref }}\left(x, t ; x_{0}\right)$ is the Green's function for the half-space system with $x>0$ and the fully reflecting wall at $x=0$. 
Using Eqs. $(4,5), C(x, t)$ can be written as

$$
C(x, t)=\int_{0}^{t} d t^{\prime} W\left(t^{\prime}\right) G_{\mathrm{ref}}\left(x, t-t^{\prime} ; 0^{+}\right),
$$

where the function $W(t)$, which equals

$$
W(t)=\int_{-\infty}^{0} d x_{0} J\left(0^{+}, t ; x_{0}\right) C\left(x_{0}, 0\right),
$$

depends on the initial and boundary conditions.

Since the subdiffusion equation is of the second order with respect to $x$, it requires two boundary conditions at the membrane. The first one assumes the continuity of the flux $J$, given by the generalized Fick's law [9], which flows through the membrane i.e. $J\left(0^{-}, t\right)=J\left(0^{+}, t\right)$. However, there is no obvious choice of the second boundary condition. Therefore, we assume that the missing condition is given by a linear combination of concentrations and flux i.e.

$$
b_{1} C\left(0^{-}, t\right)+b_{2} C\left(0^{+}, t\right)+b_{3} J(0, t)=0 .
$$

Two boundary conditions

$$
C\left(0^{+}, t\right)=\frac{1-\sigma}{1+\sigma} C\left(0^{-}, t\right)
$$

and

$$
J(0, t)=\lambda\left(C\left(0^{-}, t\right)-C\left(0^{+}, t\right)\right),
$$

discussed in [6,10] and [11], respectively, are of the general form (7). The parameters $\sigma$ and $\lambda$ control the membrane permeability $[6,10,11]$. The adopted initial condition combined with Eq. (7) provide

$$
\begin{aligned}
W(t) & =C_{0} \frac{b_{1} \sqrt{D_{\alpha}}}{b_{1}-b_{2}} \frac{1}{t^{1-\alpha / 2}} \\
& \times \sum_{k=0}^{\infty} \frac{d^{k}}{\Gamma(\alpha / 2-k(1-\alpha / 2))} \frac{1}{t^{k(1-\alpha / 2)}},
\end{aligned}
$$

where $d \equiv b_{3} \sqrt{D_{\alpha}} /\left(b_{1}-b_{2}\right)$. The Green's function $G_{\text {ref }}$, which enters Eq. (6), can be easily obtained by means of the method of images [1] as $G_{\text {ref }}\left(x, t ; x_{0}\right)=G_{0}\left(x, t ; x_{0}\right)+$ $G_{0}\left(-x, t ; x_{0}\right)$ with the known Green's function $G_{0}$ for the homogeneous system [1]. Having the explicit functions $W$ and $G_{\text {ref }}$, we write down, using Eq. (6), the concentration profile as

$$
\begin{aligned}
C(x, t) & =\int_{0}^{t} d t^{\prime} W\left(t-t^{\prime}\right) \\
& \times \frac{2}{\alpha x} H_{11}^{10}\left(\left(\frac{x}{\sqrt{D_{\alpha} t^{\prime \alpha}}}\right)^{\frac{2}{\alpha}} \mid \begin{array}{cc}
1 & 1 \\
1 & \frac{2}{\alpha}
\end{array}\right),
\end{aligned}
$$

where $H$ denotes the Fox function.

We first consider the long time approximation of the formula (11) which corresponds to the small $s$ limit of the Laplace transform $L\{f(t)\} \equiv \int_{0}^{\infty} f(t) e^{-s t}$. Taking into account only the leading contribution in the small $s$ limit, Eq. (11) gets the form

$$
C(x, t)=\frac{2 C_{0} b_{1}}{\left(b_{1}-b_{2}\right) \alpha} H_{11}^{10}\left(\left(\frac{x}{\sqrt{D_{\alpha} t^{\alpha}}}\right)^{\frac{2}{\alpha}} \mid \begin{array}{cc}
1 & 1 \\
0 & \frac{2}{\alpha}
\end{array}\right) .
$$

The solution (12) can be also obtained directly from Eq. (11), taking into account only the $k=0$ term in the expansion (10).

The series (10) can be approximated by the first term if $d \ll t^{1-\alpha / 2}$. When the boundary condition is of the form (8), the condition is trivially satisfied for any $t$ as $b_{3}=d=0$ in this case. For the boundary condition (9), we have $\lambda=b_{1} / b_{3}=-b_{2} / b_{3}$, and the long time approximation holds if

$$
\left(\frac{\sqrt{D_{\alpha}}}{2 \lambda}\right)^{\frac{1}{1-\alpha / 2}} \ll t
$$

For the membranes used in our experiments $\lambda$ is of order $10^{-2}[\mathrm{~mm} / \mathrm{s}]$ and assuming that we deal with the normal diffusion $D$ is roughly $10^{-5}\left[\mathrm{~mm}^{2} / \mathrm{s}\right]$. Thus, the l.h.s. of Eq. (13) is estimated as $2 \mathrm{~s}$. Since $10 \mathrm{~s}$ is the time step of our measurements which extend to $2500 \mathrm{~s}$, the condition (13) is fulfilled. We have also checked the condition (13) a posteriori, using the values of $\alpha$ and $D_{\alpha}$ obtained by means of our method. The 1.h.s. of Eq. (13) is again about $2 \mathrm{~s}$.

Let us now discuss the temporal evolution of nearmembrane layers in the long time approximation. Substituting the solution (12) into Eq. (2), we get the equation which simplifies to

$$
H_{11}^{10}\left(\left(\frac{\delta}{\sqrt{D_{\alpha} t^{\alpha}}}\right)^{\frac{2}{\alpha}} \mid \begin{array}{cc}
1 & 1 \\
0 & \frac{2}{\alpha}
\end{array}\right)=\frac{\kappa \alpha}{2} .
$$

One observes that Eq. (14) is solved by

$$
\delta(t)=A\left(\alpha, D_{\alpha}, \kappa\right) t^{\alpha / 2} .
$$

The near-membrane layer (15) does not depend on the parameters $b_{1}$ and $b_{2}$ while the coefficient $A$ can be recalculated into the diffusion constant $D_{\alpha}$ as

$$
D_{\alpha}=\frac{A^{2}}{\left[\left(H_{1}^{1} \frac{1}{1}\right)^{-1}\left(\frac{\alpha \kappa}{2} \mid \begin{array}{ll}
1 & 1 \\
0 & \frac{2}{\alpha}
\end{array}\right)\right]^{\alpha}} .
$$

We have also studied the near-membrane layers beyond the long time approximation using the boundary conditions (8) and (9). The condition (8) allows for the analytic treatment of Eq. (3) and the solution is of the form (12) with $2 b_{1} /\left(b_{1}-b_{2}\right)$ replaced by $1-\sigma$. Thus, the formulas derived in the long time approximation are exact for Eq (8). When Eq. (9) is used as the boundary condition, the solution of subdiffusion equation (3) for $x>0$ is 


$$
\begin{aligned}
C(x, t) & =\frac{C_{0}}{\alpha} \sum_{n=0}^{\infty}\left[-\frac{x}{2 \lambda}\left(\frac{\sqrt{D_{\alpha}}}{x}\right)^{\frac{2}{\alpha}}\right]^{n} \\
& \times H_{11}^{1}{ }_{1}\left(\left(\frac{x^{2}}{D_{\alpha} t^{\alpha}}\right)^{\frac{1}{\alpha}} \mid \begin{array}{cc}
1 & 1 \\
n\left(\frac{2}{\alpha}-1\right) & \frac{2}{\alpha}
\end{array}\right) .
\end{aligned}
$$

The solutions (12) and (17), which for normal diffusion have been discussed in $[10,11]$, qualitatively differ from each other but the differences are evident only for times which are significantly longer than those studied here.

Since the formula (17) is analytically intractable we have found the time evolution of near-membrane layer numerically. As discussed in detail in [5], we have not found any difference between the near-membrane layer obtained for the concentration profile with the boundary condition (8) and with the boundary condition (9).

Fitting the experimental $\delta(t)$ by the function $A t^{\gamma}$, we have found the index $\alpha=2 \gamma=0.90 \pm 0.01$. It does not much differ from unity but it signals subdiffusion due to the small error [5]. With the numerical values of inverse Fox functions, we recalculate the coefficient $A$ into $D_{\alpha}$ by means of the relation (16). Thus, we get $D_{0.90}=(9.8 \pm 1.0) \times 10^{-4}\left[\mathrm{~mm}^{2} / \mathrm{s}^{0.90}\right]$ for glucose and $D_{0.90}=(6.3 \pm 0.9) \times 10^{-4}\left[\mathrm{~mm}^{2} / \mathrm{s}^{0.90}\right]$ for sucrose.

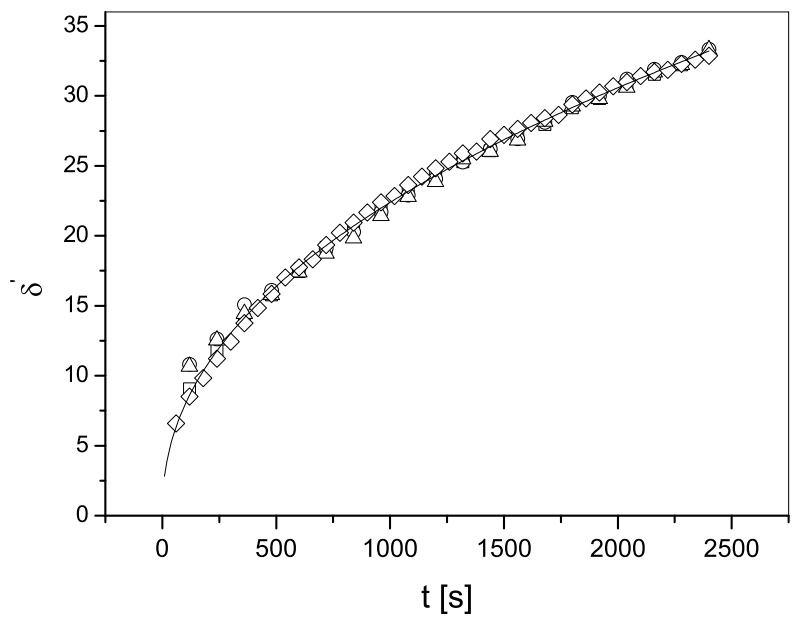

FIG. 3. The experimentally measured $\delta$ divided by the coefficient $A$ from Eq. (15). The symbols are assigned as in Fig. 2 and the line represents the function $t^{0.45}$. For clarity of the plot the error bars are not shown.

To be sure that Eq. (15), which is used to evaluate $D_{\alpha}$, properly describes the experimental $\delta(t)$, we have checked the scaling of $\delta(t)$ suggested by Eq. (15). In Fig. 3 we plot the rescaled near-membrane layer $\delta^{\prime}(t)=\delta(t) / A$, with $A$ from Eq. (15), for all values of $\kappa$, for glucose and for sucrose. The experimental points are represented as in Fig. 2. As seen, our experimental data are very well described by the function $t^{0.45}$.

Our method to determine the parameters of subdiffusion relies on the near-membrane layers. One may ask why $\alpha$ and $D_{\alpha}$ are not extracted directly form the con- centration profiles which are measured. There are three reasons to choose the near-membrane layers: experimental, theoretical and practical: 1) Measurement of $\delta$ does not suffer from the sizable $(\sim 10-15 \%)$ systematic error of absolute normalization of $C$, as only the relative concentration matters for $\delta$.2) Computed concentration profiles depend on the adopted boundary condition at a membrane while the condition is not well established even for the normal diffusion. The near-membrane layer appears to be free of this dependence. 3) When $C$ is fitted by a solution of the subdiffusion equation, there are three free parameters: $\alpha, D_{\alpha}$ and the parameter characterizing the membrane permeability. Because these fit parameters are correlated with each other it is very difficult to get their unique values. When $\delta$ is studied the membrane parameter drops out entirely, $\alpha$ is controlled by the time dependence of $\delta(t)$ while $D_{\alpha}$ is provided by the coefficient $A$.

The membrane plays only an auxiliary role in our method to measure the subdiffusion parameters but the transport in membrane systems is of interest in several fields of technology [12], where the membranes are used as filters, and biophysics [13], where the membrane transport plays a crucial role in the cell physiology. The diffusion in a membrane system is also interesting by itself as a nontrivial stochastic problem, see e.g. [10]. Thus, our study of the subdiffusion in a membrane system, which to our best knowledge has not been investigated by other authors, opens up a new field of interdisciplinary research.

[1] R. Metzler and J. Klafter, Phys. Rep. 339, 1 (2000).

[2] J.P. Bouchaud and A. Georgies, Phys. Rep. 195 (1990), 127.

[3] E. Barkai, R. Metzler, and J. Klafter, Phys. Rev. E 61, 132 (2000); E. Barkai, Phys. Rev. E 63, 046118 (2001); S. Lim and S.V. Muniandy, Phys. Rev. E 66, 021114 (2002).

[4] A. Klemm, R. Metzler, and R. Kimmich, Phys. Rev. E 65, 021112 (2002).

[5] T. Kosztołowicz, K. Dworecki, and St. Mrówczyński, [arXiv:cond-mat/0309072], submitted to Phys. Rev. E.

[6] K. Dworecki, T. Kosztołowicz, S. Wa̧sik, and St. Mrówczyński, Eur. J. Phys. E 3, 389 (2000).

[7] K. Dworecki, J. Biol. Phys. 21, 37 (1995).

[8] A. Compte, Phys. Rev. E 53, 4191 (1996).

[9] D.H. Zanette, Physica A 252, 159 (1998).

[10] T. Kosztołowicz, Phys. Rev. E 54, 3639 (1996); J. Phys. A 31, 1943 (1998); Physica A 248, 44 (1998).

[11] T. Kosztołowicz and St. Mrówczyński, Acta Phys. Pol. B 32, 217 (2001); T. Kosztołowicz, Physica A 298, 285 (2001).

[12] R. Rautenbach and R. Albert, Membrane Processes, (Wiley, Chichester, 1989).

[13] J.H.M. Thornley, Mathematical Models in Plant Physiology, (Academic Press, London, 1976). 